\title{
Relation between left ventricular function and oxidative stress in patients undergoing bypass
} surgery

\author{
E De Vecchi, M G Pala, G Di Credico, V Agape, G Paolini, P A Bonini, A Grossi, \\ R Paroni
}

\begin{abstract}
Objective-To determine whether preoperative left ventricular ejection fraction (LVEF) is related to the degree of myocardial oxidative stress during bypass surgery in man.

Design-Observational study.

Setting-Tertiary care centre.

Patients and interventions-31 patients (LVEF range was $20 \%$ to $68 \%$ ) undergoing elective coronary bypass surgery with blood cardioplegic reperfusion were studied. Arterial and coronary sinus blood was collected before aortic cross clamping (T0) and at 0 (T1), 15 (T2), and 30 (T3) minutes after unclamping. Transmural left ventricular biopsies were also obtained from 15 patients at $\mathrm{T} 0$ and at $\mathrm{T} 1$.

Main outcome measures-Glutathione and adenine nucleotides were measured in myocardial biopsies, while coronary sinus-artery differences for glutathione, nucleotides, and products of lipid peroxidation were calculated from blood specimens. Creatine kinase (myocardial band; CK-MB) was measured in plasma at four and 12 hours after operation.
\end{abstract}

Results-Myocardial glutathione and adenine nucleotides were correlated $(p<0.02)$ with preoperative LVEF both at T0 $(r=0.909$ and 0.672$)$ and T1 $(r=0.603$ and 0.605$)$. Oxidised glutathione released from the heart during reperfusion was inversely correlated with LVEF $(r=-0.448,-0.466$, and -0461 at $\mathrm{T} 1, \mathrm{~T} 2$, and $\mathrm{T} 3, \mathrm{p}<0.01$ ), while reduced glutathione ( $r=0.519$ and 0.640 at T1 and T2) and glutathione redox ratio $(r=0.647,0.714$, 0.645 , and 0.702 at T0, T1, T2, and T3) showed a direct correlation $(p<0.01)$. Lipid peroxidation at $\mathrm{T} 1$ was negatively related to LVEF ( $r=-0.492)$. CK-MB was also negatively related to LVEF $(r=-0.440$ at $4 \mathrm{~h}$ and -0.462 at $12 \mathrm{~h})$.

Conclusions-The capacity to counterbalance oxidative burst following ischaemia and reperfusion appears to be related to the functional ability of the heart.

(Heart 1998;79:242-247)

Keywords: oxidative stress; glutathione; lipid peroxidation; aortocoronary bypass

Re-establishment of blood flow in the ischaemic myocardium is a common situation in cardiovascular surgery. Although delayed re- covery to aerobic metabolism contributes to mortality and morbidity in high risk patients, ${ }^{1}$ experimental studies have shown that reperfusion itself is associated with structural and functional derangement, ${ }^{2}{ }^{3}$ thus resulting in extension of the ischaemic damage. In up to $50 \%$ of patients, ischaemia-reperfusion injury during bypass grafting is responsible for a notable depression in early postoperative myocardial function. ${ }^{4}$ Experimental ${ }^{56}$ and clinical studies ${ }^{78}$ have shown that free radicals are generated in the myocardium during coronary bypass surgery and much information is now available about the role played by oxygen free radicals in the aetiology of myocardial dysfunction after ischaemia and reperfusion. ${ }^{9}{ }^{10}$

In a recent investigation ${ }^{11}$ aimed at studying the protective activity of leucocyte depleted cardioplegia during bypass surgery, we noticed a different response to oxidative stress according to the patient's preoperative left ventricular ejection fraction (LVEF). We hypothesised that this behaviour could be caused by altered antioxidant capacity of the myocardium, and we designed the present study to verify a possible association between heart contractility (expressed as LVEF) and the capacity to counterbalance the oxidative stress occurring during myocardial revascularisation. To do this, we measured glutathione and adenine nucleotides in myocardial biopsies taken from patients undergoing bypass surgery, as well as in arterial and coronary sinus blood.

\section{Methods}

PATIENT POPULATION

The study was carried out on 31 patients admitted to hospital for elective coronary bypass operation. Left ventricular function was determined by first pass radionuclide angiography. Exclusion from the study was based on the following criteria: evolving infarction, reoperation or other comparable procedures (valve replacement or aneurysmectomy), or associated disease. Patients without viable myocardium, as determined by a positron emission tomography analysis (PET-FDG), were also excluded from the study. Preoperative data are summarised in table 1 . All patients had angina and were receiving conventional antianginal drugs (nitrates, $\beta$ blocking agents, calcium antagonists, or combinations) until the day of surgery. All the subjects gave informed consent for the study, which was approved by the medical ethics committee of our Institution. 
Table 1 Preoperative and perioperative information

\begin{tabular}{ll}
\hline Patients (n) & 31 \\
Age (years) & $66(59$ to 68$)$ \\
Sex (male/female) & $28 / 3$ \\
Previous infarction (n) & $27 / 31$ \\
Angina (CCS class, II/III/IV) & $4 / 22 / 5$ \\
Ejection fraction (\%) & $35(30$ to 50$)$ \\
Diseased vessels (n) (1/2/3) & $1 / 4 / 26$ \\
Extra-circulation circuit time (min) & $141(120$ to 162$)$ \\
Cross clamp time (min) & $89(77$ to 108$)$ \\
Distal anastomoses 0/1/2/3/4/5 (\%) & $0 / 3.3 / 16.6 / 36.6 / 30 / 13.3$ \\
Proximal anastomoses 0/1/2 (\%) & $26.6 / 50 / 23.3$ \\
Inotropic requirement (dopamine & $18 / 31$ \\
$\quad>5 \mathrm{mg} / \mathrm{kg} /$ min) (n) & \\
IABP (n) & $7 / 31$
\end{tabular}

Continuous data are expressed as median and interquartile range. The number of distal and proximal anastomosis is expressed as frequency (\%).

CCS, Canadian Cardiovascular Society system of grading angina; IABP, intra-aortic balloon pumping.

SURGICAL TECHNIQUE

A standard cardiopulmonary bypass technique was used throughout the study. Myocardial revascularisation was achieved when possible with bilateral internal mammary artery plus saphenous vein grafts. During cardiopulmonary bypass, the packed cell volume was maintained between 0.2 and 0.25 , and core temperature was $28-30^{\circ} \mathrm{C}$.

The cardiopulmonary bypass circuit was primed with Ringer's lactate solution (1500 ml), mannitol (250 ml), and Emagel (100 ml). Extracorporeal circulation was conducted with a roller pump (Stockert Instruments, Munich, Germany) and membrane oxygenator (Compact D703, Dideco, Mirandola, Italy). Blood cardioplegia was provided by a Buckberg-Shiley Plus system (Shiley Incorporated, Irvine, California, USA), which delivered oxygenated perfusion circuit blood and a crystalloid cardioplegic solution at a ratio of $4: 1$.

The physical and biochemical composition of cardioplegic solution, the time and the mode of delivery were in accordance with Buckberg's protocol. $^{12} 13$ The delivery time was divided between antegrade and retrograde through the aorta and the coronary sinus using an antegrade cannula (aortic root cannula, DLP Inch Inc, Grandrapids, Michigan, USA) and a retroplegic cannula (retroplegia coronary sinus cannula, Research Medical, Midvale, Utah, USA). Cardiac arrest was achieved with warm $\left(37^{\circ} \mathrm{C}\right)$, blood substrate enriched (aspartate, glutamate) cardioplegia delivered at a rate of 200 to $250 \mathrm{ml} / \mathrm{min}$ for five minutes, with a measured aortic root pressure not exceeding $70 \mathrm{~mm} \mathrm{Hg}$, then followed by three minutes of cold induction at the same rate. On completion of each distal anastomosis or at 20 minute intervals, cardioplegic maintenance was similarly ensured with cold blood cardioplegia in both antegrade and retrograde directions in separate ratios. Before cross clamp release, warm blood cardioplegic reperfusion was given in an antegrade-retrograde direction and through the venous graft for three minutes at a rate of 200 to $250 \mathrm{ml} / \mathrm{min}$; cardioplegic washout was performed with systemic blood in a retrograde direction for two minutes.

In order to maintain a good coronary perfusion at the end of bypass, in patients with extensive arterial grafts the mean arterial pressure was held between 70 and $90 \mathrm{~mm} \mathrm{Hg}$ by the use of inotropic drugs (dopamine). Indications for intra-aortic balloon pumping were also explored in patients with reduced LVEF in the light of our previous experience experience. $^{14} 15$

BIOPSY SPECIMENS

In 15 consecutive patient, transmural left ventricular biopsy specimens were obtained from anterolateral wall of the apex of the left ventricle with a Tru-Cut biopsy needle (Baxter, Deerfield, Illinois, USA). Necrotic or fibrotic areas were carefully avoided. Sampling occurred at the beginning of cardiopulmonary bypass (T0) and after cross clamp removal (T1); biopsies were immediately immersed in liquid nitrogen until homogenisation, which occurred within a few hours. Before analysis, frozen biopsies were quickly weighed on a microbalance and immediately homogenised $(\sim 30 \mathrm{~s})$ in $0.36 \mathrm{~mol} / 1$ cold perchloric acid (1:100, wt:vol). The probe was dipped in liquid nitrogen before each homogenization cycle and the sample taken in an ice bath. After centrifugation at $10000 \times g$ for one minute at $4^{\circ} \mathrm{C}$, the supernatant was used for glutathione and adenine nucleotides assays.

\section{BLOOD SAMPLING}

Arterial and coronary sinus blood were collected simultaneously in heparinised tubes in ice before aortic cross clamping (T0) and 0 (T1), 15 (T2), and 30 minutes (T3) after unclamping. An aliquot was immediately treated for blood measurements, and the remaining part centrifuged for plasma determinations. Lipid peroxidation was also assayed on peripheral venous plasma before induction of anaesthesia and at four and 24 hours after cardiopulmonary bypass. The myocardial band of creatine kinase (CK-MB) was measured at four and 12 hours after recovery in intensive care unit.

BIOCHEMICAL MEASUREMENTS

Total glutathione (sum of reduced, disulphide, soluble mixed disulphide, and protein bound forms) and oxidised glutathione (sum of disulphide, soluble mixed disulphide, and protein bound forms) in blood and plasma were determined as previously described. ${ }^{16}$ Reduced glutathione was calculated as the difference between total and oxidised fractions. The total soluble glutathione fraction was determined in myocardial tissue. To do this, an aliquot of the homogenate supernatant was added to $50 \mu \mathrm{l}$ of $1 \mathrm{~mol} / \mathrm{l}$ Tris buffer and $100 \mu \mathrm{l}$ of $25 \mathrm{mmol} / 1$ dithiothreitol; after 10 minutes, $350 \mu$ of 0.25 $\mathrm{mol} / 1$ perchloric acid were added, and $150 \mu \mathrm{l}$ of the mixture loaded on the high performance liquid chromatograph (HPLC) autosampler, derivatised, and injected as described before. ${ }^{17}$

Blood and plasma adenine nucleotides and hypoxanthine were measured after deproteinisation with perchloric acid. The acid supernatants from blood, plasma, and tissue homogenate were neutralised with sodium bicarbonate 
and injected on HPLC under conditions previously described. ${ }^{17}$

Lipid peroxidation in plasma was evaluated by measurement of fluorescent adducts resulting from interaction of aldehydes (end products of lipid peroxidation) with proteins, according to the method of Ward et al. ${ }^{18}$

Tissue concentrations are expressed on a gram wet weight basis. Oxidised glutathione concentrations were always expressed as GSH equivalents. Myocardial stress was assessed by the difference between coronary sinus and arterial measurements.

\section{STATISTICAL ANALYSIS}

Continuous variables are expressed as median (interquartile range). Correlation analysis was performed with Spearman's test. For myocardial biopsies, data before and after cross clamping were compared by using the Wilcoxon signed rank test. One way analysis of variance for repeated measures (Friedman's method) followed by Dunn's test was used to locate significant differences over time in blood and plasma. Statistical significance was assumed for $p<0.05$. All analyses were performed using the Sigma Stat statistical package for personal computers (Jandel $\mathrm{GmbH}$, Erkrath, Germany) running on a IBM PS/2.

\section{Results}

Preoperative status and operation details are summarised in table 1 . No patient had electrocardiographic changes and enzyme elevation suggestive of perioperative myocardial infarction or ischaemia; 15 of 18 patients who were given inotropic support had an LVEF of less than $40 \%$. One patient died the day following operation in low output, while two others died during the first month after the operation, one from a stroke and one from a fatal arrhythmia. All the patients who died had a preoperative LVEF of less than $40 \%$, which is associated with an incremental risk of perioperative death. ${ }^{19} 20$

MYOCARDIAL TISSUE

Already at T0, myocardial content of soluble glutathione appeared strictly correlated with preoperative LVEF, increasing with the improvement in heart function $(r=0.909$, $\mathrm{p}<0.001$ ) (fig 1). At T1, the tissue content of glutathione was 183 (104 to 215 ) $\mathrm{nmol} / \mathrm{g}$, significantly reduced from the T0 value of 321 (271 to 553$) \mathrm{nmol} / \mathrm{g}(\mathrm{p}<0.001)$. Although not as strong as before surgery, the correlation with LVEF was still good $(r=0.603, \mathrm{p}<0.02)$ (fig 1). Similar behaviour was shown by intracellular adenosine triphosphate (ATP), which was 248 (77 to 414 ) $\mathrm{nmol} / \mathrm{g}$ at $\mathrm{T} 0$, decreasing to $127(53$ to 182$) \mathrm{nmol} / \mathrm{g}$ at $\mathrm{T} 1(\mathrm{p}<0.01, \mathrm{~T} 1 v$ T0). When the results were analysed according to the patient's preoperative LVEF ( $<40 \%$ in nine patients; $\geqslant 40 \%$ in six patients), the myocardial ATP was 108 (56 to 222) v 394 (273 to $558) \mathrm{nmol} / \mathrm{g}$ in the basal state, and 66 (33 to 126) v 208 (139 to 127$) \mathrm{nmol} / \mathrm{g}$ at unclamping $(\mathrm{p}<0.01$, low $v$ normal LVEF). The adenosine diphosphate (ADP) content was also significantly different in the two groups, both at

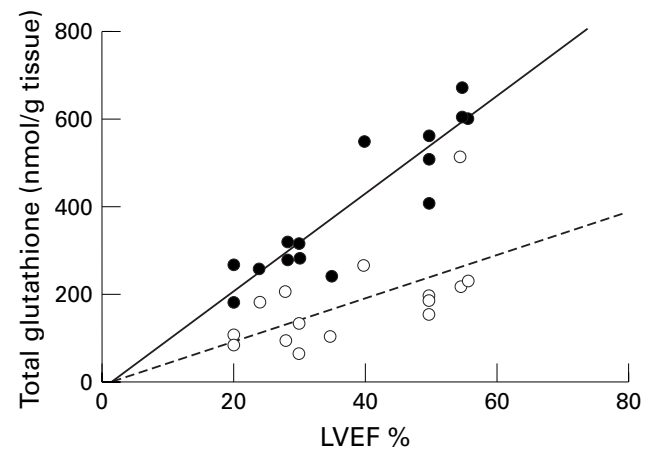

Figure 1 Relation between myocardial total soluble glutathione content and heart function (LVEF). •, basal measurement $(r=0.909, p<0.001) ; 0$, immediately after cross clamp removal $(r=0.603, p<0.02)$.

T0 (319 (220 to 413$) v 1260$ (764 to 1473$)$ $\mathrm{nmol} / \mathrm{g}$ ) and at T1 (202 (95 to 246) $v 699$ (384 to 930$) \mathrm{nmol} / \mathrm{g}$ ). Adenosine monophosphate did not change over time: 524 (359 to 871) and 326 (281 to 608) at T1 and T0, respectively. The sum of high energy adenine nucleotides (ATP+ADP+AMP), which was 1.45 (0.79 to $1.22) \mu \mathrm{mol} / \mathrm{g}$ at $\mathrm{T} 0$, and decreased to 0.79 (0.58 to 1.39$) \mu \mathrm{mol} / \mathrm{g}$ at $\mathrm{T} 1$ ( $\mathrm{p}<0.01)$, significantly correlated with LVEF both at T0 and T1 $(r=0.672$ and 0.605 , respectively $\mathrm{p}<0.02)$.

PLASMA

As expected, at T0 no evident coronary artery difference was detectable. At T1 a significant release of total glutathione was observed, with a trend to normalisation in the following 30 minutes (table 2). The observed modifications were mainly due to the oxidised forms which sharply increased at $\mathrm{T} 1$ and remained significantly altered in the following 30 minutes (table 2). Release of reduced glutathione after unclamping, although detectable, never reached significance (table 2). If patients were stratified according to their preoperative LVEF, it became evident that glutathione released from the heart was mainly the oxidised form in patients with an LVEF of $<40 \%$, while in patients with an LVEF of $\geqslant 40 \%$ there was a predominance of the reduced form. This resulted in a significant decrease in redox ratio at T1 $v$ T0 in subjects with depressed function (0.16 (0.09 to 0.30$) \quad v \quad 0.30 \quad(0.23 ; 0.38)$, $\mathrm{p}<0.01$ ), while no alterations were observed in patients with $\mathrm{LVEF} \geqslant 40 \%$ (0.59 (0.43 to $0.71) v 0.45$ (0.39 to 0.54$)$ ). Plasma redox ratio was positively correlated with LVEF after unclamping $(r=0.714,0.645$, and 0.702 at $\mathrm{T} 1, \mathrm{~T} 2$, and $\mathrm{T} 3$ ), and even before the cross clamp period $(r=0.674, \mathrm{p}<0.01$ ) (fig 2$)$.

As a byproduct of the degradation of myocardial high energy phosphates, a sustained release of hypoxanthine occurred at unclamping, and this was correlated with worsening LVEF ( $r=-0.440$ at T0).

Release of lipid peroxidation products at unclamping was also found to be correlated with LVEF $(r=-0.492)$. Sustained lipid peroxidation was evident at peripheral venous level at three hours (91 (82 to 119) fluorescence units (UF)/ml) and 24 hours after bypass (110 (77 to 143$) \mathrm{UF} / \mathrm{ml}$ ) $v$ baseline (53 (36 to 
Table 2 Transmyocardial oxidative stress in plasma

\begin{tabular}{|c|c|c|c|c|}
\hline & TO & $T 1$ & $T 2$ & T3 \\
\hline Total glutathione $(\mu \mathrm{mol} / 1)$ & $-0.13(-1.14$ to 0.56$)$ & $2.70^{\star}(1.67$ to 3.94$)$ & $1.48^{\star}(1.13$ to 2.50$)$ & $0.83(-0.22$ to 1.46$)$ \\
\hline Oxidised glutathione $(\mu \mathrm{mol} / \mathrm{l})$ & $0.21(-0.17$ to 0.44$)$ & $1.88^{\star}(1.00$ to 2.67$)$ & $0.98^{\star}(0.66$ to 1.70$)$ & $0.57^{\star}(0.24$ to 0.88$)$ \\
\hline Reduced glutathione $(\mu \mathrm{mol} / \mathrm{l})$ & $-0.05(-0.27$ to 0.30$)$ & $0.50(-0.25$ to 1.28$)$ & $0.70(-0.05$ to 1.13$)$ & $0.50(-0.02$ to 0.98$)$ \\
\hline Redox ratio & $0.36(0.26$ to 0.45$)$ & $0.33(0.14$ to 0.56$)$ & $0.35(0.26$ to 0.55$)$ & $0.31(0.20$ to 0.52$)$ \\
\hline Hypoxanthine $(\mu \mathrm{mol} / \mathrm{l})$ & $0.08(-0.23$ to 0.71$)$ & $4.58^{\star}(2.94$ to 6.42$)$ & $4.25^{\star}(2.51$ to 7.35$)$ & $3.12^{\star}(1.33$ to 5.74$)$ \\
\hline
\end{tabular}

Data are expressed as median (interquartile range) of coronary sinus-coronary artery differences.

${ }^{\star} \mathrm{p}<0.05 v$ T0 value.

T0, before aortic cross clamp; T1/T2/T3, 0/15/30 minutes after unclamping; redox ratio, reduced to total oxidised glutathione ratio.
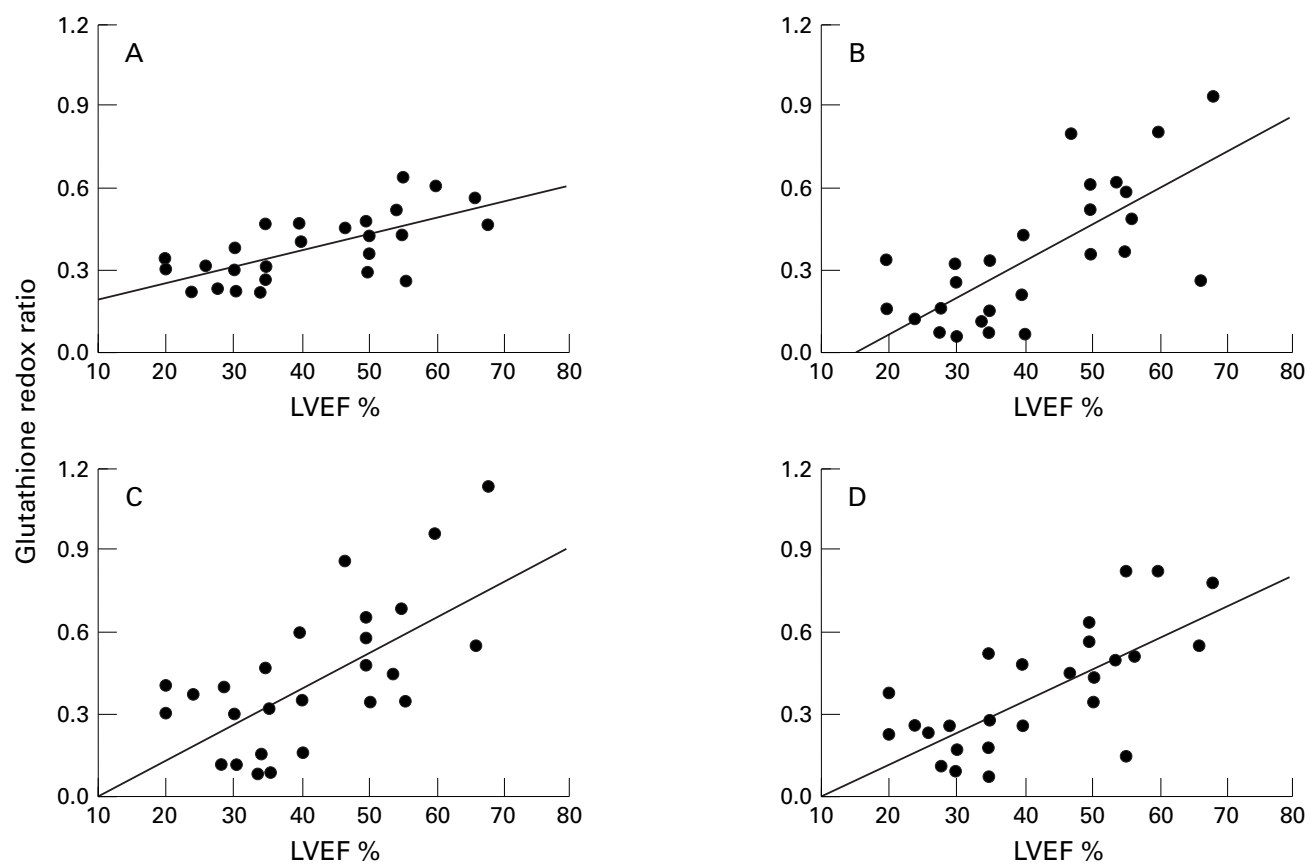

Figure 2 Relation between coronary plasma glutathione redox ratio and heart function (LVEF): (A) before aortic cross clamping $(r=0.674, p<0.01)$; (B) immediately after cross clamp removal $(r=0.714, p<0.01)$; $(C) 15$ minutes after unclamping $(r=0.645, p<0.01)$; (D) 30 minutes after unclamping $(r=0.702, p<0.01)$.

82) $\mathrm{UF} / \mathrm{ml}$ ), the extension of the phenomenon being correlated with preoperative LVEF $(r=-0.384$ and -0.440 at 3 and 24 hours, respectively).

No patient had CK-MB values suggestive of perioperative infarction: 22 (14 to 32$) \mu \mathrm{g} / 1$ at 4 hours and 21 (15 to 31$) \mu \mathrm{g} / 1$ at 24 hours after bypass. At both these times the values were negatively correlated with LVEF ( $r=-0.440$ and $-0.462, \mathrm{p}<0.02)$. Patients with depressed LVEF (<40\%) showed more evident CK-MB release than patients with normal LVEF both at 4 hours (27.5 (22.0 to 34.0) v 15.5 (10.7 to $20.0) \mu \mathrm{g} / \mathrm{l}$ ) and 12 hours (30 (22.5 to 40.8) v $15(11.6$ to 15.4$) \mu \mathrm{g} / \mathrm{l})$. An association between CK-MB release at 4 hours and plasma oxidised glutathione at $\mathrm{T} 1$ was also found $(r=0.400)$.

BLOOD

Coronary sinus-coronary artery differences for erythrocyte oxidised glutathione (table 3) were inversely correlated with LVEF $(r=-0.480)$. The increment in oxidised glutathione was responsible for a significant decrease in the redox ratio, which remained depressed in subsequent observations (table 3 ).

Significant formation of hypoxanthine was observed at $\mathrm{T} 1$. This remained present at $\mathrm{T} 2$ and T3 (table 3 ) and showed a negative corre- lation with LVEF $(r=-0.478,-0.463$, and -0.390 at 0,15 , and 30 minutes).

\section{Discussion}

Oxygen free radicals and their metabolites play an important role in the pathophysiology of ischaemia-reperfusion injury. Experimental studies using electron paramagnetic spin resonance techniques have identified and quantified free radical species in ischaemic and reperfused hearts of dogs and rabbits. ${ }^{21}{ }^{22}$ However, direct measurement of free radicals in humans is difficult because of their transient nature and the complexity of the available techniques. Evidence for free radical production during ischaemia and reperfusion has been obtained by determining concentrations of antioxidants such as glutathione ${ }^{723}$ and vitamin $\mathrm{E}^{24}$ or lipid peroxidation products. ${ }^{85}$ The ejection fraction has been considered for many years to be a risk factor for cardiac surgery, ${ }^{19}{ }^{20}$ although because of the recent discovery of hibernating myocardium it does not now seem to impose a serious limitation on surgery. However, to the best of our knowledge few studies have investigated whether differences in contractility are linked to a different antioxidant capacity, and therefore to a major susceptibility to oxidative insult. Our present study was aimed at resolving this 
Table 3 Transmyocardial oxidative stress in blood

\begin{tabular}{|c|c|c|c|c|}
\hline & TO & $T 1$ & $T 2$ & $T 3$ \\
\hline Oxidised glutathione (nmol/g Hb) & $1.00(-4.75$ to 9.00$)$ & $7.00(-10$ to 30$)$ & $6.00(-7.00$ to 3.00$)$ & $-2.00(-12$ to 9.25$)$ \\
\hline Redox ratio & $132(116$ to 174$)$ & $96^{\star}(50$ to 115$)$ & $96^{\star}(79$ to 123$)$ & $105^{\star}(85$ to 144$)$ \\
\hline Hypoxanthine ( $\mu \mathrm{mol} / 1 \mathrm{RBC})$ & $0.00(-1.48$ to 2.27$)$ & $7.00^{\star}(4.00$ to 12$)$ & $7.00^{\star}(5.00$ to 18$)$ & $7.00^{\star}(1.88$ to 14$)$ \\
\hline
\end{tabular}

Data are expressed as median (interquartile range) of coronary sinus-coronary artery differences.

${ }^{\star} \mathrm{p}<0.05 v \mathrm{~T} 0$ value.

$\mathrm{T} 0$, before aortic cross clamp; T1/T2/T3, 0/15/30 minutes after unclamping; redox ratio, reduced to total oxidised glutathione ratio.

issue by evaluating free radical induced alterations in myocardial tissue during bypass surgery.

We found that myocardial glutathione content and plasma redox ratio were positively correlated with heart function even under basal conditions, thus suggesting the involvement of free radicals in the genesis and development of ischaemic heart disease. As previously proposed, ${ }^{7}$ chronic ischaemia is not only responsible for a severe impairment of myocardial contractility, but it can also promote a loss of tissue glutathione and scavenging enzyme activity (glutathione peroxidase), thus resulting in a lack of defence against free radical toxicity. Prolonged ischaemia is responsible for a decrease in myocardial non-protein sulphydryl groups - mainly glutathione - and in high energy phosphates, and this becomes a rate limiting factor for detoxification of oxygen species. ${ }^{19}{ }^{20}$ The final effect could be a vicious circle in which chronic ischaemia impairs LVEF and reduces natural scavenger availability, thus producing a low ability to counterbalance the damage of ischaemia and worsening myocardial contractility.

Our data on biopsies indicate that the cross clamp period produces a massive loss of myocardial glutathione. Reoxygenation on reperfusion results in oxidative damage, as evidenced by release of the disulphide form of glutathione in the coronary sinus. The degree of release was related to preoperative heart function, so only in patients with the worst LVEF was the plasma glutathione redox ratio altered. At removal of cross clamping, reduced glutathione was also released from the myocardium; this release was greater in patients with good heart function, so in these subjects no significant change in coronary redox ratio was detected during the operation. It may be hypothesised that in these patients the antioxidant systems were more effective or that there was a greater availability of reduced glutathione, as described by others for chronic heart failure. ${ }^{26} 27$

Levels of tissue adenine nucleotides decrease during the cross clamp period with subsequent washout of hypoxanthine, thus confirming other published data. ${ }^{8}$ During the reperfusion period, however, myocardial release slowly diminished, suggesting a gradual recovery of cellular energetics. Although the role of xanthine oxidoreductase activity ${ }^{28}$ in the heart is of little importance, release of hypoxanthine from the myocardium can trigger generation of oxygen free radicals in the systemic circulation, especially in patients whose antioxidant defences are depressed. Moreover, the higher ATP content of normal myocardium-even under basal conditions-suggests a greater availability of energy in the patients with higher LVEF values, thus making them more able to repair oxidative damage. Our data on lipid peroxidation and $\mathrm{CK}-\mathrm{MB}$ further confirm the hypothesis that depressed heart function favours sustained free radical activity and consequently greater lipid peroxidation. The correlation between CK-MB release and oxidised glutathione release indicates a link between oxidative stress and cell injury, as already reported. $^{729}$

The occurrence of oxidative stress during bypass surgery was also clearly detected in blood, and the direct linkage between heart function and the erythrocyte redox ratio after unclamping supports the hypothesis that in patients with poor LVEF the erythrocytes have to counterbalance a major production of oxidative species.

The measured variables did not correlate with cross clamp time. Ferrari and coworkers, using a crystalloid cardioplegia, reported a positive correlation between cross clamp time and oxidised glutathione release for clamping periods longer than 30 minutes, while the correlation was not evident for periods less than 30 minutes. ${ }^{7}$ We suppose that the blood cardioplegia employed in this study is probably protective enough to make oxidative stress independent of the duration of the clamping period.

In conclusion, our data support the hypothesis that cardiac function and free radical activity are linked, and that the ability to counteract ischaemia-reperfusion induced oxidative damage is related to heart function.

We gratefully acknowledge Dr Cecilia Gobbi (Biomedical Statistics Unit, IRCCS H San Raffaele, Milan, Italy) for her helpful suggestions for statistical analysis of data. This work was partially supported by a grant from National Research Council, partially supported by a grant from
Italy (grant No 94.02819 CT04).

1 Berger RL, Davis KB, Kaiser GC. Preservation of the myocardium during coronary artery bypass grafting. Circulation 1981;64:61-6.

2 Jennings RB, Reimer KA. Factors involved in salvaging ischemic myocardium. Effects of reperfusion of arterial ischemic myocardium. Effects of
blood. Circulation 1983;68:125-36.

3 Becker LC, Ambrosio G. Myocardial consequence of reperBecker LC, Ambrosio G. Myocardial consequen
fusion. Prog Cardiovasc Dis 1987;30:23-44.

4 Reduto LA, Lawrie GM, Reid JW, et al. Sequential postoperative assessment of left ventricular performance with gated blood pool imaging following aortocoronary bypass surgery. Am Heart f 1981;101:59-66.

5 Simpson PJ, Lucchesi BR. Free radicals and myocardial ischemia and reperfusion injury. F Lab Clin Med 1987;110: 13-30.

6 Bolli R. Mechanism of myocardial "stunning". Circulation 1990;82:723-38.

7 Ferrari R, Alfieri O, Curello S, et al Occurrence of oxidative stress during reperfusion of the human heart. Circulation 1990;81:201-11.

8 Lazzarino G, Raatikainen P, Nuutinen M, et al Myocardial release of malondialdehyde and purine compounds during coronary bypass surgery. Circulation 1994;90:291-7.

9 McCord JM. Oxygen derived free radicals in postischemic tissue injury. $N$ Engl f Med 1985;312:159-63.

10 Kukreja RC, Hess ML. The oxygen free radical system: from equations through membrane-protein interactions to cardiovascular injury and protection. Cardiovasc Res 1992;
26:641-55. 
11 De Vecchi E, Paroni R, Pala MG, et al. Role of leucocytes in free radical production during myocardial revascularisafree radical production during
tion. Heart 1997;77:449-55.

12 Buckberg GD. Strategies and logic of cardioplegic delivery to prevent, avoid and preserve ischemic and reperfusion damage. F Thorac Cardiovasc Surg 1987;93:127-39.

13 Buckberg GD. Antegrade and retrograde blood cardioplegia to ensure cardioplegic distribution: operative techniques and objectives. F Thorac Cardiovasc Surg 1989;4:216-38.

14 Biagioli B, Giomarelli PP, Gnudi G, et al. Myocardial function in early hours after coronary artery by-pass grafting: comparison of two cardioplegic methods. Ann Thorac Surg 1993;56:1315-23.

15 Paolini G, Zuccari M, Mariani MA, et al. Myocardial revascularization in patients with severe ischaemic left ventricular dysfunction: a clinical experience. Cardiovasc Surg 1994;2:88-92.

16 Paroni R, De Vecchi E, Cighetti G, et al. Total, oxidized and protein bound glutathione in blood, plasma and tissue protein bound glutathione in blood, plasma and tissue 52 .

17 Paroni R, De Vecchi E, Lubatti L, et al. Influence of the 21-aminosteroid U74389F on ischemia-reperfusion injury in the rat. Eur F Pharmacol 1995;294:737-42.

18 Ward PA, Till GO, Hatherill JR, et al. Systemic complement activation, lung injury, and products of lipid peroxidation. $\mathscr{f}$ Clin Invest 1985;76:517-27.

19 Alderman EL, Fisher LD, Litwin P, et al. Results of coronary artery surgery in patients with poor left ventricular function (CASS). Circulation 1983;68:785-95.

20 Hochberg MS, Parsomet V, Gulchinsky I, et al. Coronary artery bypass grafting in patients with ejection fraction below forty percent. F Thorac Cardiovasc Surg 1983;86: 519-27.
21 Zweier JL, Flaherty JT, Weisfeldt ML. Direct measurement of free radical generation following reperfusion of ischemic myocardium. Proc Natl Acad Sci USA 1987;84:1404-7.

22 Bolli R, Patel BS, Jeroudi MO, et al. Demonstration of free radical generation in "stunned" myocardium of intact dogs with the use of the spin trap $\alpha$ phenyl N-tert-butyl nitrone. 7 Clin Invest 1988;82:476-85.

23 Mezzetti A, Calafiore AM, Lapenna D, et al. Intermittent antegrade warm cardioplegia reduces oxidative stress and improves metabolism of the ischemic reperfused human improves metabolism of the ischemic reperfused human
myocardium. F Thorac Cardiovasc Surg 1995;109:787-95.

24 Coghlan JG, Flitter WD, Clutton SM, et al. Lipid peroxidation and changes in vitamin $\mathrm{E}$ levels during coronary artery bypass grafting. I Thorac Cardiovasc Surg 1993;106:26874 .

25 Coghlan JG, Flitter WD, Clutton SM, et al. Allopurinol pretreatment improves postoperative recovery and reduces lipid peroxidation in patients undergoing coronary artery lipid peroxidation in patients undergoing coronary artery 56.

26 McMurray J, McLay J, Chopra M, et al. Evidence for enhanced free radical activity in chronic congestive heart failure secondary to coronary artery disease. Am 7 Cardiol 1990;65:1261-2.

27 McMurray J, Chopra M, Abdullah I, et al. Evidence of oxidative stress in chronic heart failure in humans. Eur Heart 7. 1993;14:1493-8.

28 Huizer T, de Wong JW, Nelson JA, et al. Urate production by human heart. $\mathcal{F}$ Mol Cell Cardiol 1989;21:691-5.

29 Curello S, Ceconi C, de Giuli F, et al. Oxidative stress during reperfusion of human hearts: potential sources of oxygen free radicals. Cardiovasc Res 1995;29:118-25. 